Indian J. Pure Appl. Math., 51(4): 1673-1695, December 2020

(c) Indian National Science Academy

DOI: $10.1007 / \mathrm{s} 13226-020-0489-2$

\title{
HOPF BIFURCATION AND STABILITY SWITCHES INDUCED BY HUMORAL IMMUNE DELAY IN HEPATITIS C
}

\author{
Sonjoy Pan and Siddhartha P. Chakrabarty \\ Department of Mathematics, Indian Institute of Technology Guwahati, \\ Guwahati 781 039, Assam, India \\ e-mails: sonjoy.pan@iitg.ac.in; pratim@iitg.ac.in
}

(Received 25 February 2019; accepted 8 November 2019)

\begin{abstract}
The role of humoral immune delay on the dynamics of $\mathrm{HCV}$ infection incorporating both the modes of infection transmission, namely, viral and cellular transmissions with a non-cytolytic cure of infected hepatocytes is studied. The local and global asymptotic stability of the boundary equilibria, namely, infection-free and immune-free equilibrium are analyzed theoretically as well as numerically under the conditions on the basic reproduction number and the humoral immune reproduction number. The existence of Hopf bifurcation and consequent occurrence of bifurcating periodic orbits around the humoral immune activated equilibrium are illustrated. The findings show that Hopf bifurcation and stability switches occur under certain conditions as the bifurcation parameter crosses the critical values. Furthermore, the dynamical effect of the development rate of $B$ cells is investigated numerically. The results obtained show that the system becomes unstable from stable and regains stability from instability depending on the development rate of $B$ cells for a fixed delay value. Further, the results suggest that a high antigenic stimulation in humoral immunity is beneficial for uninfected hepatocytes with a significant reduction in virions density.
\end{abstract}

Key words : Humoral immunity; cellular transmission; Hopf bifurcation; stability switch.

2010 Mathematics Subject Classification : 92B05, 92D30.

\section{INTRODUCTION}

An estimated 71 million individuals are affected worldwide by chronic hepatitis $\mathrm{C}$ virus (HCV) infection (a blood-borne hepatological condition) resulting in about 399 thousand cases of fatalities happening in 2016, due to liver cirrhosis and hepatocellular carcinoma [1]. Among HCV infected individuals $60-80 \%$ of the cases become chronic, out of which about $15-30 \%$ face the risk of developing 
liver cirrhosis in the long run [1]. The transmission of HCV primarily happens through unscreened blood transfusions, injecting drug use and inadequately sterilized medical equipment [2]. HCV, which is a positive single-stranded viral RNA genome from the family of Flaviviridae [3], replicates very rapidly resulting in difficulty in developing a vaccine for HCV [4]. The pioneering in-vivo model for HCV dynamics [5] based on similar models for human immunodeficiency virus (HIV) and hepatitis $B$ virus (HBV) dynamics, incorporated the interferon- $\alpha$ (IFN- $\alpha$ ) antiviral therapy for HCV infection with three model populations, namely, uninfected and infected hepatocytes, and virions. The analysis in [5] demonstrated that the antiviral therapy is more effective in the reduction of HCV RNA load with the efficacy increasing with an increase in the dosage of IFN- $\alpha$, accompanied by a minor effect in blocking of production of infected hepatocytes. Dixit et al. [6] included ribavirin along with IFN in the antiviral therapy protocol and described the effect of this combination therapy in HCV infection. Due to the action of ribavirin, a fraction of the virions become non-infectious and hence are not involved in the production of infected hepatocytes. The analysis [6] demonstrated that the ribavirin in combination with IFN significantly improves the process of HCV RNA decline, but as a monotherapy, it produces only short-term early response in terms of decrease of viral load. The extended models $[7,8]$ of the models in [5, 6] included the argument of homeostatic mechanism by which liver can regenerate itself, thereby which the hepatocytes can proliferate up to a certain maximum level. A comparison of the extended models $[7,8]$ with the models in $[5,6]$ showed that the analysis of these models $[5,6]$ exhibits a biphasic decline in viral load (a rapid viral decline followed by a constant level of viral load), while the models in [7, 8] can better predict the kinetics of HCV RNA load in chronic stage and also explain the biphasic as well as triphasic viral decay. A fast viral decline in the third phase was observed in cases where the majority of hepatocytes were already infected before the initiation of antiviral therapy [8]. The effectiveness of pegylated IFN and ribavirin as antiviral therapy for HCV infection was analyzed by estimating the clearance rate of HCV RNA and the infected cells as well [9].

The role of cytotoxic T lymphocyte (CTL) and humoral immune response in HCV infection was investigated through the mathematical modeling in $[10,11]$. The role of CTL is to reduce the HCV infection with the antibody playing a role of neutralizing HCV RNA. The analysis found the correlation between the CTL and antibody when both are activated during chronic HCV infection. It was observed that the infection would be asymptotic if the CTL activates strongly, but persistent leading to pathology in case of weak CTL response, even in presence of antibody. A complex mechanism of the immune system for $\mathrm{HCV}$ patients was considered in the model formulation of [12] which includes the role of dendritic cell (DC) with CTL response in such a way that CTL is produced through the 
cross-presentation of activated DC and decays by a direct presentation of infected cells. It was also observed that the activation of immune response is dependent on initial DC and CTL levels. The consideration of cure of infected cells through the non-cytolytic process in which a part of the infected cells get converted to uninfected cells, was introduced in [13, 14].

Timpe et al. [15] observed that the mode of HCV transmission can not only be virus-to-cell but also cell-to-cell. A HCV model considering cell-to-cell transmission was studied for the optimal antiviral treatment policy in [16]. Further, from the epidemiological point of view, the consideration of intracellular delay (the time needed for the hepatocytes to be infected or for replication of the virions) and immune delay (the time needed for antigenic stimulation for the development of $B$ cells in case of antibody response or for the development of $T$ cells in case of CTL response) are more realistic when analyzing HCV viral dynamics. The intracellular delay was incorporated in a HCV model in [17] which included the activation of CTL and antibody response. The role of the humoral immune delay in a HBV model with two stages of infected cells, namely, latently infected and actively infected was illustrated in [18]. A general viral dynamics model with the effect of the humoral immune delay was analyzed in [19], where the existence of Hopf bifurcation was observed. The effect of the various intracellular delays on the dynamics of other viral infections (like HBV, HIV infection), incorporating both virus-to-cell and cell-to-cell transmissions, was studied in [20-22]. A general viral infection model considering the viral as well as cellular transmission of infection with cell-mediated immune response was proposed and analyzed with the effect of the intracellular delay as well as cellmediated immune delay [23]. The results showed that the delays could lead to stability switches and occurrence of bifurcating periodic solution, depending upon the intrinsic rate of logistic growth and infection transmission rate as well.

The limited number of models in the literature which illustrated the effect of humoral immune response in viral dynamics did not include either cell-to-cell transmission or cure of infected cells. On the other hand, the models which considered cell-to-cell transmission did not take into account the humoral immune delay as well as cure of infected cells. However, in this work, we include all these factors in the proposed model.

\section{Mathematical Model}

The dynamics of HCV infection with both viral and cellular transmissions and cure rate in the presence of humoral immune response was analyzed in [24]. This model assumed that subsequent to the entry of the virions, the humoral immune response is stimulated to instantaneously generate $B$ cells. However, from a realistic epidemiological point of view, there is a time delay between the initial vi- 
ral entry into the liver and subsequent process of antigenic stimulation to generate $B$ cells $[19,25]$. However, it is considered that the antibody neutralizing the virions is immediate [19]. Accordingly, we incorporate the humoral immune delay to propose the delay model as follows:

$$
\begin{aligned}
\frac{d T(t)}{d t} & =\lambda-\beta_{1} T(t) V(t)-\beta_{2} T(t) I(t)-d_{1} T(t)+\alpha I(t), \\
\frac{d I(t)}{d t} & =\beta_{1} T(t) V(t)+\beta_{2} T(t) I(t)-d_{2} I(t)-\alpha I(t), \\
\frac{d V(t)}{d t} & =k I(t)-d_{3} V(t)-p V(t) Z(t), \\
\frac{d Z(t)}{d t} & =c V(t-\tau) Z(t-\tau)-d_{4} Z(t) .
\end{aligned}
$$

Here $T(t), I(t), V(t)$ and $Z(t)$ represent the densities of the uninfected hepatocytes, actively infected hepatocytes, virions and humoral immunity ( $B$ cell or antibody) at time $t$, respectively. It is assumed that the uninfected hepatocytes are being sourced at a constant rate $\lambda$ (within the liver) and are cleared at a natural death rate of $d_{1}$. It is also considered that the virions and the infected hepatocytes infect the healthy hepatocytes at a rate of $\beta_{1}$ and $\beta_{2}$ respectively. The infected hepatocytes have a natural death rate of $d_{2}$. Due to the non-cytolytic process, the infected hepatocytes are converted to uninfected ones at a rate $\alpha$. The infected hepatocytes abet the production of free virions at a rate $k$, which in turn decay at a rate of $d_{3}$. Subsequent to entry of the virions into the body, the humoral immune response stimulates itself to generate $B$ cell at a rate $c$, which results in the $B$ cells being neutralized at a rate $p . B$ cells are cleared at a natural death rate of $d_{4}$. Finally, $\tau$ represents the time needed for antigenic stimulation for developing humoral immunity, i.e., the humoral immune response at time $t$ depends on the populations of $B$ cells at time $(t-\tau)$. From biological considerations, we consider all the model parameters to be positive. Accordingly, the system (2.1) is subject to the initial condition taken in the form

$$
\begin{aligned}
& T(\theta)=\psi_{1}(\theta), I(\theta)=\psi_{2}(\theta), V(\theta)=\psi_{3}(\theta), Z(\theta)=\psi_{4}(\theta), \\
& \psi_{i}(\theta) \geq 0, \theta \in[-\tau, 0], \psi_{i}(0)>0, i=1,2,3,4
\end{aligned}
$$

where $\left(\psi_{1}(\theta), \psi_{2}(\theta), \psi_{3}(\theta), \psi_{4}(\theta)\right) \in \mathbb{C}\left([-\tau, 0], \mathbb{R}_{+0}^{4}\right)$, the Banach space of continuous functions mapping the interval $[-\tau, 0]$ into $\mathbb{R}_{+0}$ with norm $\|\psi\|=\sup _{-\tau \leq \theta \leq 0}\left\{\left|\psi_{i}(\theta)\right|, i=1,2,3,4\right\}$, where $\mathbb{R}_{+0}^{4}=\left\{\left(x_{1}, x_{2}, x_{3}, x_{4}\right) \mid x_{i} \geq 0, i=1,2,3,4\right\}$.

The uniqueness and positivity of the solution to the system (2.1) with the initial condition (2.2) can be proved using the theory of functional differential equations [26], and by Theorem A.4 in [27] and Lemma 2 in [28], respectively. Further, the boundedness of the solution can be proved on the lines of [24]. 
The model system (2.1) has three equilibria, namely [24],

1. The infection-free equilibrium, $E_{0}=\left(T_{0}, I_{0}, V_{0}, Z_{0}\right)=\left(\frac{\lambda}{d_{1}}, 0,0,0\right)$.

2. The immune-free equilibrium, $E_{1}=\left(T_{1}, I_{1}, V_{1}, Z_{1}\right)$, where $T_{1}=\frac{d_{3}\left(d_{2}+\alpha\right)}{\beta_{1} k+\beta_{2} d_{3}}$, $I_{1}=\frac{d_{1} T_{1}}{d_{2}}\left[\frac{\lambda\left(\beta_{1} k+\beta_{2} d_{3}\right)}{d_{1} d_{3}\left(d_{2}+\alpha\right)}-1\right], V_{1}=\frac{k}{d_{3}} I_{1}, Z_{1}=0$.

3. The humoral immune activated equilibrium, $E^{*}=\left(T^{*}, I^{*}, V^{*}, Z^{*}\right)$, where

$$
T^{*}=\frac{\left(d_{2}+\alpha\right) I^{*}}{\beta_{2} V^{*}+\beta_{2} I^{*}}, I^{*}=\frac{-m_{2}+\sqrt{m_{2}^{2}+4 m_{1} m_{3}}}{2 m_{1}}, V^{*}=\frac{d_{4}}{c}, Z^{*}=\frac{d_{3}}{p}\left(\frac{c k}{d_{3} d_{4}} I^{*}-1\right)
$$

with $m_{1}=\beta_{2} c d_{2}, m_{2}=\beta_{1} d_{2} d_{4}+c d_{1}\left(d_{2}+\alpha\right)-\lambda \beta_{2} c, m_{3}=\lambda \beta_{1} d_{4}$.

Further, the system (2.1) has two reproduction numbers, namely [24], the basic reproduction number, $\quad R_{0}=\frac{\lambda\left(\beta_{1} k+\beta_{2} d_{3}\right)}{d_{1} d_{3}\left(d_{2}+\alpha\right)}$, and the humoral immune reproduction number, $R_{H}=\frac{c k \lambda\left(\beta_{1} k+\beta_{2} d_{3}\right)}{c k d_{1} d_{3}\left(d_{2}+\alpha\right)+d_{2} d_{3} d_{4}\left(\beta_{1} k+\beta_{2} d_{3}\right)}$. Clearly, $R_{H}<R_{0}$. Further $\frac{c k}{d_{3} d_{4}} I^{*}>1$ if $R_{H}>1$. It is obvious that the infection-free equilibrium $\left(E_{0}\right)$ exists unconditionally, the immune-free equilibrium ( $\left.E_{1}\right)$ exists if $R_{0}>1$ and the humoral immune activated equilibrium $\left(E^{*}\right)$ exists if $R_{H}>1$.

\subsection{Stabilities of Boundary Equilibria}

Theorem 1 - The infection-free equilibrium $E_{0}$ is locally as well as globally asymptotically stable for any $\tau>0$ if $R_{0}<1$ and unstable if $R_{0}>1$.

PROOF : The proof for local stability easily follows from the Routh-Hurwitz criteria.

Further, the global stability can be proved using the Lyapunov-LaSalle invariance principle [26] by choosing the Lyapunov functional,

$$
\begin{aligned}
L_{1}(t)= & T_{0} g\left(\frac{T}{T_{0}}\right)+I(t)+\frac{\beta_{1} T_{0}}{d_{3}} V(t)+\frac{\beta_{1} p T_{0}}{c d_{3}} Z(t)+\frac{\alpha}{2\left(d_{1}+d_{2}\right) T_{0}} \\
& {\left[T(t)-T_{0}+I(t)\right]^{2}+\frac{\beta_{1} p T_{0}}{d_{3}} \int_{t-\tau}^{t} V(\xi) Z(\xi) d \xi }
\end{aligned}
$$

where $g(x)=x-1-\ln (x), x>0$.

Theorem 2 - The immune-free equilibrium $E_{1}$ is locally asymptotically stable if $R_{H}<1<R_{0}$ and globally asymptotically stable if $R_{H} \leq 1<R_{0} \leq 1+\frac{d_{2}}{\alpha}$, for any $\tau>0$. Further, $E_{1}$ is unstable if $R_{H}>1$.

PROOF : The characteristic equation obtained by linearizing (2.1) at $E_{1}$ is given by

$$
\left(x+d_{4}-c V_{1} e^{-x \tau}\right)\left(x^{3}+B_{1} x^{2}+B_{2} x+B_{3}\right)=0,
$$


where

$$
\begin{aligned}
& B_{1}=d_{1}+d_{3}+\beta_{1} V_{1}+\beta_{2} I_{1}+\frac{\beta_{1} k T_{1}}{d_{3}} \\
& B_{2}=d_{1} d_{3}+\left(d_{2}+d_{3}\right)\left(\beta_{1} V_{1}+\beta_{2} I_{1}\right)+\frac{d_{1} \beta_{1} k T_{1}}{d_{3}}, \\
& B_{3}=d_{2} d_{3}\left(\beta_{1} V_{1}+\beta_{2} I_{1}\right) .
\end{aligned}
$$

All the three roots of the equation $x^{3}+B_{1} x^{2}+B_{2} x+B_{3}=0$ have negative real parts if $R_{0}>1$, which has been proved in [24]. We now investigate whether any complex root with positive real part exists for the following equation:

$$
x+d_{4}-c V_{1} e^{-x \tau}=0 .
$$

Let $x=a+i b(i=\sqrt{-1}, a \geq 0)$ be a root of (2.3). Substituting $x=a+i b(i=\sqrt{-1}, a \geq 0)$ in (2.3) and separating the real and imaginary parts, we obtain the following:

$$
\begin{aligned}
a+d_{4}-c V_{1} e^{-a \tau} \cos (b \tau) & =0, \\
b+c V_{1} e^{-a \tau} \sin (b \tau) & =0 .
\end{aligned}
$$

Equations (2.4) and (2.5) give

$$
\begin{aligned}
b^{2}= & c^{2} V_{1}^{2} e^{-2 a \tau}-\left(a+d_{4}\right)^{2}=\left[d_{4} e^{-a \tau}-d_{4}-a-\frac{\lambda c k e^{-a \tau}\left(1-R_{H}\right)}{d_{2} d_{3} R_{H}}\right] \\
& {\left[d_{4}+a+c V_{1} e^{-a \tau}\right]<0 \text { if } R_{H}<1, }
\end{aligned}
$$

which is a contradiction. Therefore every root of (2.3) must have negative real part. Hence, $E_{1}$ is locally asymptotically stable for any $\tau>0$ if $R_{H}<1<R_{0}$.

Further, let $F(x)=x+d_{4}-c V_{1} e^{-x \tau}$. Observe that $F(0)=d_{4}-c V_{1}=\frac{\lambda c k}{d_{2} d_{3} R_{H}}\left(1-R_{H}\right)<0$ if $R_{H}>1$ and $\lim _{x \rightarrow+\infty} F(x)=+\infty$. Since $F(x)$ is continuous on $(-\infty, \infty)$, therefore by the intermediate value property, it follows that the equation $F(x)=0$ has at least one positive real root. Hence the characteristic equation at $E_{1}$ has at least one positive real root. Thus $E_{1}$ is unstable if $R_{H}>1$.

Further, the global stability can be proved using the the Lyapunov-LaSalle invariance principle [26] by choosing the Lyapunov functional,

$$
\begin{aligned}
L_{2}(t)= & T_{1} g\left(\frac{T}{T_{1}}\right)+I_{1} g\left(\frac{I}{I_{1}}\right)+\frac{\beta_{1} T_{1} V_{1}^{2}}{k I_{1}} g\left(\frac{V}{V_{1}}\right)+\frac{\beta_{1} p T_{1} V_{1}}{c k I_{1}} Z \\
& +\frac{\alpha}{2\left(d_{1}+d_{2}\right) T_{1}}\left[\left(T-T_{1}\right)+\left(I-I_{1}\right)\right]^{2}+\frac{\beta_{1} p T_{1} V_{1}}{k I_{1}} \int_{t-\tau}^{t} V(\xi) Z(\xi) d \xi
\end{aligned}
$$


where $g(x)=x-1-\ln (x), x>0$.

\subsection{The Interior Equilibrium and Hopf Bifurcation}

The characteristic equation obtained by linearizing (2.1) at $E^{*}$ is given by

$$
x^{4}+a_{1} x^{3}+a_{2} x^{2}+a_{3} x+a_{4}-c V^{*}\left(x^{3}+b_{1} x^{2}+b_{2} x+b_{3}\right) e^{-x \tau}=0,
$$

where

$$
\begin{aligned}
& a_{1}=d_{1}+d_{4}+\beta_{1} V^{*}+\beta_{2} I^{*}+\frac{k I^{*}}{V^{*}}+\frac{\beta_{1} T^{*} V^{*}}{I^{*}} \\
& a_{2}=\left(d_{1}+\beta_{1} V^{*}+\beta_{2} I^{*}\right)\left(d_{4}+\frac{k I^{*}}{V^{*}}\right)+d_{2}\left(\beta_{1} V^{*}+\beta_{2} I^{*}\right)+\left(d_{1}+d_{4}\right) \frac{\beta_{1} T^{*} V^{*}}{I^{*}}+\frac{d_{4} k I^{*}}{V^{*}} \\
& a_{3}=\left(d_{1}+\beta_{1} V^{*}+\beta_{2} I^{*}\right) \frac{d_{4} k I^{*}}{V^{*}}+d_{2}\left(\beta_{1} V^{*}+\beta_{2} I^{*}\right)\left(d_{4}+\frac{k I^{*}}{V^{*}}\right)+d_{1} d_{4}\left(\frac{\beta_{1} T^{*} V^{*}}{I^{*}}\right) \\
& a_{4}=d_{2} d_{4}\left(\beta_{1} V^{*}+\beta_{2} I^{*}\right) \frac{k I^{*}}{V^{*}}, \\
& b_{1}=d_{1}+d_{3}+\beta_{1} V^{*}+\beta_{2} I^{*}+\frac{\beta_{1} T^{*} V^{*}}{I^{*}} \\
& b_{2}=d_{1} d_{3}+\left(d_{2}+d_{3}\right)\left(\beta_{1} V^{*}+\beta_{2} I^{*}\right)+\left(d_{1}-p Z^{*}\right) \frac{\beta_{1} T^{*} V^{*}}{I^{*}} \\
& b_{3}=d_{2} d_{3}\left(\beta_{1} V^{*}+\beta_{2} I^{*}\right)-\left(\frac{\beta_{1} T^{*} V^{*}}{I^{*}}\right) d_{1} p Z^{*} .
\end{aligned}
$$

When $\tau>0,(2.6)$ becomes transcendental and therefore some of the roots may cross the imaginary axis to the right. We now investigate the existence of purely imaginary roots of (2.6). Substituting $x=i \omega(i=\sqrt{-1}, \omega>0)$ in (2.6) and separating the real and imaginary parts, we obtain the following,

$$
\begin{aligned}
\omega^{4}-a_{2} \omega^{2}+a_{4} & =c V^{*}\left(-b_{1} \omega^{2}+b_{3}\right) \cos (\omega \tau)+c V^{*}\left(-\omega^{3}+b_{2} \omega\right) \sin (\omega \tau), \\
-a_{1} \omega^{3}+a_{3} \omega & =c V^{*}\left(-\omega^{3}+b_{2} \omega\right) \cos (\omega \tau)-c V^{*}\left(-b_{1} \omega^{2}+b_{3}\right) \sin (\omega \tau) .
\end{aligned}
$$

Squaring and adding the equations (2.8) and (2.9), we obtain

$$
\omega^{8}+P_{1} \omega^{6}+P_{2} \omega^{4}+P_{3} \omega^{2}+P_{4}=0
$$

where

$$
\begin{aligned}
P_{1} & =a_{1}^{2}-2 a_{2}-c^{2} V^{* 2}, \\
P_{2} & =a_{2}^{2}-2 a_{1} a_{3}+2 a_{4}-b_{1}^{2} c^{2} V^{* 2}+2 b_{2} c^{2} V^{* 2}, \\
P_{3} & =a_{3}^{2}-2 a_{2} a_{4}+2 b_{1} b_{3} c^{2} V^{* 2}-b_{2}^{2} c^{2} V^{* 2}, \\
P_{4} & =a_{4}^{2}-b_{3}^{2} c^{2} V^{* 2} .
\end{aligned}
$$


Let $\gamma=\omega^{2}$. Then (2.10) becomes

$$
G(\gamma) \equiv \gamma^{4}+P_{1} \gamma^{3}+P_{2} \gamma^{2}+P_{3} \gamma+P_{4}=0
$$

Therefore (2.6) has a pair of purely imaginary roots $\pm i \omega$ if and only if (2.11) has a positive real root $\omega^{2}$. If (2.6) has no positive real root, then (2.11) has no purely imaginary root, in which case the existence of Hopf bifurcation is ruled out and hence $E^{*}$ is locally asymptotically stable for any $\tau>0$. Now, we suppose that (2.11) has $m(1 \leq m \leq 4)$ positive roots, say, $\gamma_{n}, n=1,2, \ldots, m$. Then (2.10) has $m$ positive roots, say, $\omega_{n}=\sqrt{\gamma_{n}}, n=1,2, \ldots, m$.

Solving (2.8) and (2.9) for $\cos (\omega \tau)$, we obtain

$$
\cos (\omega \tau)=\frac{\left(\omega^{4}-a_{2} \omega^{2}+a_{4}\right)\left(-b_{1} \omega^{2}+b_{3}\right)+\left(-a_{1} \omega^{3}+a_{3} \omega\right)\left(-\omega^{3}+b_{2} \omega\right)}{c V^{*}\left[\left(b_{1} \omega^{2}-b_{3}\right)^{2}+\left(\omega^{3}-b_{2} \omega\right)^{2}\right]} .
$$

When $\omega=\omega_{n}(n=1,2, \ldots, m)$, we obtain the following from (2.12),

$\tau=\tau_{n}^{(j)}=\frac{1}{\omega_{n}} \arccos \left[\frac{\left(\omega_{n}^{4}-a_{2} \omega_{n}^{2}+a_{4}\right)\left(-b_{1} \omega_{n}^{2}+b_{3}\right)+\left(-a_{1} \omega_{n}^{3}+a_{3} \omega_{n}\right)\left(-\omega_{n}^{3}+b_{2} \omega_{n}\right)}{c V^{*}\left[\left(b_{1} \omega_{n}^{2}-b_{3}\right)^{2}+\left(\omega_{n}^{3}-b_{2} \omega_{n}\right)^{2}\right]}\right]+\frac{2 j \pi}{\omega_{n}}$,

where $n=1,2, \ldots, m$ and $j=0,1,2, \ldots$ Hence (2.6) has a pair of purely imaginary roots $\pm i \omega_{n}$ with $\tau=\tau_{n}^{(j)}$. Further, note that $\left\{\tau_{n}^{(j)}\right\}$ is a monotonically increasing sequence for every $n=$ $1,2, \ldots, m$ and $\lim _{j \rightarrow \infty} \tau_{n}^{(j)}=\infty$. Therefore there exists a $n_{0} \in\{1,2, \ldots, m\}$ such that

$$
\tau_{n_{0}}^{(0)}=\min \left\{\tau_{n}^{(j)} \mid n=1,2, \ldots, m ; j=0,1,2, \ldots\right\} .
$$

Denote

$$
\tau_{0}=\tau_{n_{0}}^{(0)}, \omega_{0}=\omega_{n_{0}} \text { and } \gamma_{0}=\gamma_{n_{0}}
$$

Since $E^{*}$ is locally asymptotically stable for $\tau=0$ if $R_{H}>1$ [24]. Therefore, by Butler's Lemma [31], $E^{*}$ remains locally asymptotically stable for $\tau<\tau_{0}$ if $R_{H}>1$. Let $x(\tau)=\xi(\tau)+i \omega(\tau)$ be a root of (2.6) near $\tau=\tau_{0}$ with $\xi\left(\tau_{0}\right)=0, \omega\left(\tau_{0}\right)=\omega_{0}$. Therefore to prove the transversality condition for the existence of Hopf bifurcation [32] at $\tau=\tau_{0}$, we establish the following Lemma.

Lemma $1-\left[\frac{d \operatorname{Re}(x)}{d \tau}\right]_{\tau=\tau_{0}}$ and $G^{\prime}\left(\omega_{0}^{2}\right)$ have same sign, provided $G^{\prime}\left(\omega_{0}^{2}\right) \neq 0$.

PROOF : Differentiating (2.6) with respect to $\tau$, we obtain

$$
\begin{aligned}
& {\left[\left(4 x^{3}+3 a_{1} x^{2}+2 a_{2} x+a_{3}\right)-c V^{*} e^{-x \tau}\left(3 x^{2}+2 b_{1} x+b_{2}\right)+c \tau V^{*} e^{-x \tau}\right.} \\
& \left.\left(x^{3}+b_{1} x^{2}+b_{2} x+b_{3}\right)\right] \frac{d x}{d \tau}=-\left(x^{3}+b_{1} x^{2}+b_{2} x+b_{3}\right) c V^{*} e^{-x \tau} x .
\end{aligned}
$$


This implies

$$
\begin{aligned}
{\left[\frac{d x}{d \tau}\right]^{-1} } & =-\frac{4 x^{3}+3 a_{1} x^{2}+2 a_{2} x+a_{3}}{c V^{*} x e^{-x \tau}\left(x^{3}+b_{1} x^{2}+b_{2} x+b_{3}\right)}+\frac{3 x^{2}+2 b_{1} x+b_{2}}{x\left(x^{3}+b_{1} x^{2}+b_{2} x+b_{3}\right)}-\frac{\tau}{x} \\
& =-\frac{4 x^{3}+3 a_{1} x^{2}+2 a_{2} x+a_{3}}{x\left(x^{4}+a_{1} x^{3}+a_{2} x^{2}+a_{3} x+a_{4}\right)}+\frac{3 x^{2}+2 b_{1} x+b_{2}}{x\left(x^{3}+b_{1} x^{2}+b_{2} x+b_{3}\right)}-\frac{\tau}{x} .
\end{aligned}
$$

Substituting $x=i \omega_{0}$, we obtain

$$
\left[\frac{d x}{d \tau}\right]_{\tau=\tau_{0}}^{-1}=-\frac{\left(-3 a_{1} \omega_{0}^{2}+a_{3}\right)+i\left(-4 \omega_{0}^{3}+2 a_{2} \omega_{0}\right)}{\omega_{0}\left[a_{1} \omega_{0}^{3}-a_{3} \omega_{0}+i\left(\omega_{0}^{4}-a_{2} \omega_{0}^{2}+a_{4}\right)\right]}+\frac{\left(-3 \omega_{0}^{2}+b_{2}\right)+i\left(2 b_{1} \omega_{0}\right)}{\omega_{0}\left[\omega_{0}^{3}-b_{2} \omega_{0}+i\left(-b_{1} \omega_{0}^{2}+b_{3}\right)\right]}-\frac{\tau}{i \omega_{0}} .
$$

Therefore

$$
\begin{aligned}
\operatorname{Re}\left[\frac{d x}{d \tau}\right]_{\tau=\tau_{0}}^{-1}= & -\frac{\left(a_{3}-3 a_{1} \omega_{0}^{2}\right)\left(a_{1} \omega_{0}^{2}-a_{3}\right)+\left(2 a_{2}-4 \omega_{0}^{2}\right)\left(\omega_{0}^{4}-a_{2} \omega_{0}^{2}+a_{4}\right)}{\left(a_{1} \omega_{0}^{3}-a_{3} \omega_{0}\right)^{2}+\left(\omega_{0}^{4}-a_{2} \omega_{0}^{2}+a_{4}\right)^{2}} \\
& +\frac{\left(b_{2}-3 \omega_{0}^{2}\right)\left(\omega_{0}^{2}-b_{2}\right)+2 b_{1}\left(b_{3}-b_{1} \omega_{0}^{2}\right)}{\left(\omega_{0}^{3}-b_{2} \omega_{0}\right)^{2}+\left(b_{3}-b_{1} \omega_{0}^{2}\right)^{2}} .
\end{aligned}
$$

Using (2.8) and (2.9), we obtain

$$
\begin{aligned}
& \operatorname{Re}\left[\frac{d x}{d \tau}\right]_{\tau=\tau_{0}}^{-1} \\
= & \frac{-\left(a_{3}-3 a_{1} \omega_{0}^{2}\right)\left(a_{1} \omega_{0}^{2}-a_{3}\right)-\left(2 a_{2}-4 \omega_{0}^{2}\right)\left(\omega_{0}^{4}-a_{2} \omega_{0}^{2}+a_{4}\right)+c^{2} V^{* 2}\left[\left(b_{2}-3 \omega_{0}^{2}\right)\left(\omega_{0}^{2}-b_{2}\right)+2 b_{1}\left(b_{3}-b_{1} \omega_{0}^{2}\right)\right]}{c^{2} V^{* 2}\left[\left(b_{3}-b_{1} \omega_{0}^{2}\right)^{2}+\left(\omega_{0}^{3}-b_{2} \omega_{0}\right)^{2}\right]} .
\end{aligned}
$$

This upon simplification becomes

$$
\operatorname{Re}\left[\frac{d x}{d \tau}\right]_{\tau=\tau_{0}}^{-1}=\frac{4 \omega_{0}^{6}+3 \omega_{0}^{4} P_{1}+2 \omega_{0}^{2} P_{2}+P_{3}}{c^{2} V^{* 2}\left[\left(b_{3}-b_{1} \omega_{0}^{2}\right)^{2}+\left(\omega_{0}^{3}-b_{2} \omega_{0}\right)^{2}\right]} .
$$

Hence

$$
\operatorname{Re}\left[\frac{d x}{d \tau}\right]_{\tau=\tau_{0}}^{-1}=\frac{G^{\prime}\left(\omega_{0}^{2}\right)}{c^{2} V^{* 2}\left[\left(b_{3}-b_{1} \omega_{0}^{2}\right)^{2}+\left(\omega_{0}^{3}-b_{2} \omega_{0}\right)^{2}\right]}
$$

Thus

$$
\operatorname{sign}\left\{\left[\frac{d R e(x)}{d \tau}\right]_{\tau=\tau_{0}}\right\}=\operatorname{sign}\left\{\operatorname{Re}\left[\frac{d x}{d \tau}\right]_{\tau=\tau_{0}}^{-1}\right\}=\operatorname{sign}\left\{G^{\prime}\left(\omega_{0}^{2}\right)\right\} .
$$

Thus the result about the existence of Hopf bifurcation is stated in the following theorem: 
Theorem 3 - Suppose $R_{H}>1$. Then

(i) The interior equilibrium $E^{*}$ is locally asymptotically stable for any $\tau>0$, if (2.11) has no positive real root.

(ii) The interior equilibrium $E^{*}$ is locally asymptotically stable for $\tau \in\left(0, \tau_{0}\right)$, if (2.11) has at least one positive real root.

(iii) The system (2.1) undergoes a Hopf bifurcation from the interior equilibrium $E^{*}$ as $\tau$ crosses the critical value $\tau_{0}$, if $\gamma_{0}$ is a simple root of (2.11), where

$$
\tau_{0}=\frac{1}{\omega_{0}} \arccos \left[\frac{\left(\omega_{0}^{4}-a_{2} \omega_{0}^{2}+a_{4}\right)\left(-b_{1} \omega_{0}^{2}+b_{3}\right)+\left(-a_{1} \omega_{0}^{3}+a_{3} \omega_{0}\right)\left(-\omega_{0}^{3}+b_{2} \omega_{0}\right)}{c V^{*}\left[\left(b_{1} \omega_{0}^{2}-b_{3}\right)^{2}+\left(\omega_{0}^{3}-b_{2} \omega_{0}\right)^{2}\right]}\right] .
$$

PROOF : (i) This case has already been proved in the preceding discussion.

(ii) By the definition of $\tau_{0},(2.11)$ has no positive real roots for $\tau \in\left(0, \tau_{0}\right)$. Hence all the roots of (2.6) have negative real parts. Thus $E^{*}$ is locally asymptotically stable for $\tau \in\left(0, \tau_{0}\right)$.

(iii) Suppose $\gamma_{0}$ is a simple root of (2.11). Then we have $G^{\prime}\left(\omega_{0}^{2}\right) \neq 0$. If $G^{\prime}\left(\omega_{0}^{2}\right)<0$, then (2.6) has at least one root with positive real part when $\tau$ is slightly less than $\tau_{0}$, which contradicts conclusion (ii) of Theorem 3. Therefore, we have $G^{\prime}\left(\omega_{0}^{2}\right)>0$. Hence there exists a Hopf bifurcation for the system (2.1) when $\tau$ crosses the critical value $\tau_{0}$.

Next, we determine the conditions in terms of the model parameters, for which Hopf bifurcation occurs around the interior equilibrium $E^{*}$. Accordingly, we define,

$$
\begin{aligned}
& u_{1}=\frac{1}{2} P_{2}-\frac{3}{16} P_{1}^{2}, \quad u_{2}=\frac{1}{32} P_{1}^{3}-\frac{1}{8} P_{1} P_{2}+P_{3}, \\
& \Delta=\left(\frac{u_{2}}{2}\right)^{2}+\left(\frac{u_{1}}{3}\right)^{3}, \epsilon=-\frac{1}{2}+\frac{\sqrt{3}}{2} i, \quad i=\sqrt{-1}, \\
& v_{1}=\left(-\frac{u_{2}}{2}+\sqrt{\Delta}\right)^{\frac{1}{3}}+\left(-\frac{u_{2}}{2}-\sqrt{\Delta}\right)^{\frac{1}{3}}, \\
& v_{2}=\left(-\frac{u_{2}}{2}+\epsilon \sqrt{\Delta}\right)^{\frac{1}{3}}+\left(-\frac{u_{2}}{2}-\epsilon^{2} \sqrt{\Delta}\right)^{\frac{1}{3}}, \\
& v_{3}=\left(-\frac{u_{2}}{2}+\epsilon^{2} \sqrt{\Delta}\right)^{\frac{1}{3}}+\left(-\frac{u_{2}}{2}-\epsilon \sqrt{\Delta}\right)^{\frac{1}{3}}, \\
& y_{j}=v_{j}-\frac{3}{4} P_{1}, j=1,2,3 .
\end{aligned}
$$

Therefore, using Lemma 2.1 and Lemma 2.2 of [33] and Lemma 3.1 of [34], we can restate Theorem 3 as follows: 
Theorem 4 - Suppose $R_{1}>1$ with $\tau_{0}$ and $\omega_{0}$ being already defined in (2.14). Then

(i) The humoral immune activated equilibrium $E^{*}$ is locally asymptotically stable for any $\tau>0$, if $P_{4} \geq 0$ and one of the following conditions is satisfied:

(a) $\Delta>0$ and $y_{1}<0$.

(b) $\Delta=0$ and $y_{2}<0$.

(c) $\Delta<0$ and $y_{3}<0$.

(ii) The humoral immune activated equilibrium $E^{*}$ is locally asymptotically stable for $\tau \in$ $\left(0, \tau_{0}\right)$, if one of the following conditions is satisfied:

(a) $P_{4}<0$

(b) $P_{4} \geq 0, \Delta \geq 0, y_{1}>0$ and $G\left(y_{1}\right)<0$.

(c) $P_{4} \geq 0, \Delta<0$ and there exists at least one $y \in\left\{y_{1}, y_{2}, y_{3}\right\}$ such that $y>0$ and $G(y) \leq 0$.

(iii) The system (2.1) undergoes a Hopf bifurcation (leading to bifurcating periodic orbits) from the humoral immune activated equilibrium $E^{*}$ when $\tau$ crosses the critical value $\tau_{0}$ provided $G^{\prime}\left(\omega_{0}^{2}\right)>$ 0.

\section{Estimation of the Maximum Length of Delay to Preserve Stability}

In the previous section, the existence of bifurcating periodic orbits was investigated. The occurrence of the periodic orbits in a small neighborhood of $\tau_{0}$ happens either for $\tau<\tau_{0}$ or $\tau>\tau_{0}$. In order to analyze the stability of the periodic orbits, we estimate (following the approach in [35]) the maximum length of the delay to preserve the stability of the bifurcating limit cycle.

Let $P(t)=T(t)-T^{*}, Q(t)=I(t)-I^{*}, R(t)=V(t)-V^{*}, S(t)=Z(t)-Z^{*}$. Linearizing (2.1) about the interior equilibrium point $E^{*}\left(T^{*}, I^{*}, V^{*}, Z^{*}\right)$, we obtain

$$
\begin{aligned}
\frac{d P(t)}{d t} & =\left(-\beta_{1} V^{*}-\beta_{2} I^{*}-d_{1}\right) P(t)+\left(\alpha-\beta_{2} T^{*}\right) Q(t)-\beta_{1} T^{*} R(t), \\
\frac{d Q(t)}{d t} & =\left(\beta_{1} V^{*}+\beta_{2} I^{*}\right) P(t)+\left(\beta_{2} T^{*}-d_{2}-\alpha\right) Q(t)+\beta_{1} T^{*} R(t), \\
\frac{d R(t)}{d t} & =k Q(t)-\left(d_{3}+p Z^{*}\right) R(t)-p V^{*} S(t), \\
\frac{d S(t)}{d t} & =c Z^{*} R(t-\tau)+c V^{*} S(t-\tau)-d_{4} S(t) .
\end{aligned}
$$


Taking Laplace transform of (3.1), we obtain

$$
\begin{aligned}
\left(s+\beta_{1} V^{*}+\beta_{2} I^{*}+d_{1}\right) \bar{P}(s) & =\left(\alpha-\beta_{2} T^{*}\right) \bar{Q}(s)-\beta_{1} T^{*} \bar{R}(s)+P(0), \\
\left(s+d_{2}+\alpha-\beta_{2} T^{*}\right) \bar{Q}(s) & =\left(\beta_{1} V^{*}+\beta_{2} I^{*}\right) \bar{P}(s)+\beta_{1} T^{*} \bar{R}(s)+Q(0), \\
\left(s+d_{3}+p Z^{*}\right) \bar{R}(s) & =k \bar{Q}(s)-p V^{*} \bar{S}(s)+R(0), \\
\left(s+d_{4}\right) \bar{S}(s) & =c Z^{*} e^{-s \tau} \bar{R}(s) \\
& +c V^{*} e^{-s \tau} \bar{S}(s)+S(0)+c Z^{*} e^{-s \tau} K_{1}(s)+c V^{*} e^{-s \tau} K_{2}(s),
\end{aligned}
$$

where

$$
K_{1}(s)=\int_{-\tau}^{0} e^{-s t} R(t) d t, K_{2}(s)=\int_{-\tau}^{0} e^{-s t} S(t) d t
$$

and $\bar{P}(s), \bar{Q}(s), \bar{R}(s), \bar{S}(s)$ are the Laplace transforms of $P(t), Q(t), R(t), S(t)$ respectively.

Combining all the equations of (3.2) and using (2.7), we obtain,

$$
\begin{aligned}
& {\left[s^{4}+a_{1} s^{3}+a_{2} s^{2}+a_{3} s+a_{4}-c V^{*} e^{-s \tau}\left(s^{3}+b_{1} s^{2}+b_{2} s+b_{3}\right)\right] \bar{P}(s) } \\
= & {\left[s^{3}+\left(\frac{K^{*} I^{*}}{V^{*}}+\frac{\beta_{1} T^{*} V^{*}}{I^{*}}+d_{4}-c V^{*} e^{-s \tau}\right) s^{2}\right.} \\
& +\left\{\left(\frac{k I^{*}}{V^{*}}+\frac{\beta_{1} T^{*} V^{*}}{I^{*}}\right)\left(d_{4}-c V^{*} e^{-s \tau}\right)+c p V^{*} Z^{*} e^{-s \tau}\right\} s \\
& \left.+\frac{\beta_{1} c p T^{*} V^{* 2} Z^{*}}{I^{*}} e^{-s \tau}\right] P(0) \\
& +\left[\left(\alpha-\beta_{2} T^{*}\right) s^{2}+\left\{\left(\alpha-\beta_{2} T^{*}\right)\left(\frac{k I^{*}}{V^{*}}+d_{4}-c V^{*} e^{-s \tau}\right)-k \beta_{1} T^{*}\right\} s\right. \\
& \left.-\frac{d_{2} d_{4} k I^{*}}{V^{*}}+\left\{d_{2} k I^{*}+\left(\alpha-\beta_{2} T^{*}\right) p V^{*} Z^{*}\right\} c e^{-s \tau}\right] Q(0) \\
& -\left[s^{2}+\left(d_{2}+d_{4}-c V^{*} e^{-s \tau}\right) s+d_{2}\left(d_{4}-c V^{*} e^{-s \tau}\right)\right] \beta_{1} T^{*} R(0) \\
& +\left(s+d_{2}\right) \beta_{1} p T^{*} V^{*} S(0)+c Z^{*} e^{-s \tau} K_{1}(s)+c V^{*} e^{-s \tau} K_{2}(s) .
\end{aligned}
$$

A necessary and sufficient condition for $E^{*}$ to be locally asymptotically stable is that all the poles of $\bar{P}(s)$ must have negative real parts [35]. Therefore, by using the Nyquist criterion [36], we obtain the sufficient conditions for local asymptotic stability of $E^{*}$ as follows

$$
\begin{aligned}
\operatorname{ImH} H\left(i \eta_{0}\right) & >0 \\
\operatorname{ReH}\left(i \eta_{0}\right) & =0
\end{aligned}
$$

where $H(s)=s^{4}+a_{1} s^{3}+a_{2} s^{2}+a_{3} s+a_{4}-c V^{*} e^{-s \tau}\left(s^{3}+b_{1} s^{2}+b_{2} s+b_{3}\right)$, and $\eta_{0}$ is the smallest 
positive root of $\operatorname{Re} H\left(i \eta_{0}\right)=0$, which satisfies $\operatorname{ImH}\left(i \eta_{0}\right)>0$ as well. Now, (3.3) and (3.4) give

$$
\begin{aligned}
-a_{1} \eta_{0}^{3}+a_{3} \eta_{0}+c V^{*}\left(\eta_{0}^{3}-b_{2} \eta_{0}\right) \cos \left(\eta_{0} \tau\right)-c V^{*}\left(b_{1} \eta_{0}^{2}-b_{3}\right) \sin \left(\eta_{0} \tau\right) & >0 \\
\eta_{0}^{4}-a_{2} \eta_{0}^{2}+a_{4}+c V^{*}\left(b_{1} \eta_{0}^{2}-b_{3}\right) \cos \left(\eta_{0} \tau\right)+c V^{*}\left(\eta_{0}^{3}-b_{2} \eta_{0}\right) \sin \left(\eta_{0} \tau\right) & =0
\end{aligned}
$$

Therefore the sufficient condition for stability of $E^{*}$ is that (3.5) and (3.6) hold simultaneously. In order to estimate the length of the delay to preserve the stability of $E^{*}$, we have to find an upper bound of $\eta_{0}$ (independent of $\tau$ ). From (3.6), we have,

$$
\eta_{0}^{4}=a_{2} \eta_{0}^{2}-a_{4}-c V^{*}\left(b_{1} \eta_{0}^{2}-b_{3}\right) \cos \left(\eta_{0} \tau\right)-c V^{*}\left(\eta_{0}^{3}-b_{2} \eta_{0}\right) \sin \left(\eta_{0} \tau\right)
$$

Using the bounds $\left|\sin \left(\eta_{0} \tau\right)\right| \leq 1$ and $\left|\cos \left(\eta_{0} \tau\right)\right| \leq 1$, we obtain from (3.7),

$$
\eta_{0}^{4}-c V^{*} \eta_{0}^{3}-\left(a_{2}+c V^{*}\left|b_{1}\right|\right) \eta_{0}^{2}-c V^{*}\left|b_{2}\right| \eta_{0}-a_{4}-c V^{*}\left|b_{3}\right| \leq 0
$$

Let $\eta^{+}$be the smallest positive root of (3.8) when the equality holds. Then $\eta_{0} \leq \eta^{+}$. Inequality (3.5) can be written as

$$
a_{2} \eta_{0}^{2}<\frac{a_{2} a_{3}}{a_{1}}+\frac{a_{2} c V^{*}}{a_{1}}\left(\eta_{0}^{2}-b_{2}\right) \cos \left(\eta_{0} \tau\right)-\frac{a_{2} c V^{*}}{a_{1} \eta_{0}}\left(b_{1} \eta_{0}^{2}-b_{3}\right) \sin \left(\eta_{0} \tau\right)
$$

Adding (3.6) and (3.9), we obtain,

$$
\begin{aligned}
& c V^{*}\left[\eta_{0}^{3}+\left(\frac{a_{2} b_{1}}{a_{1}}-b_{2}\right) \eta_{0}-\frac{a_{2} b_{3}}{a_{1} \eta_{0}}\right] \sin \left(\eta_{0} \tau\right)+c V^{*}\left[\left(b_{1}-\frac{a_{2}}{a_{1}}\right) \eta_{0}^{2}+\left(\frac{a_{2} b_{2}}{a_{1}}-b_{3}\right)\right] \\
& {\left[1-\cos \left(\eta_{0} \tau\right)\right]<\frac{a_{2} a_{3}}{a_{1}}-a_{4}+c V^{*}\left(\frac{a_{2} b_{2}}{a_{1}}-b_{3}\right)+c V^{*}\left(b_{1}-\frac{a_{2}}{a_{1}}\right) \eta_{0}^{2}-\eta_{0}^{4} .}
\end{aligned}
$$

We have $\sin \left(\eta_{0} \tau\right) \leq \eta^{+} \tau$ and $1-\cos \left(\eta_{0} \tau\right)=2 \sin ^{2}\left(\frac{\eta_{0} \tau}{2}\right) \leq \frac{\eta^{{ }^{2}} \tau^{2}}{2}$. We now suppose that $M_{1} \tau^{2}+M_{2} \tau<M_{3}$ holds, where

$$
\begin{aligned}
& M_{1}=\frac{c V^{*}}{2}\left(\left|b_{1}-\frac{a_{2}}{a_{1}}\right| \eta^{+^{4}}+\left|\frac{a_{2} b_{2}}{a_{1}}-b_{3}\right| \eta^{+^{2}}\right) \\
& M_{2}=c V^{*}\left(\eta^{+^{4}}+\left|\frac{a_{2} b_{1}}{a_{1}}-b_{2}\right| \eta^{+^{2}}+\frac{a_{2} b_{3}}{a_{1}}\right), \\
& M_{3}=a_{4}+\frac{a_{2} a_{3}}{a_{1}}+c V^{*}\left|\frac{a_{2} b_{2}}{a_{1}}-b_{3}\right|+c V^{*}\left|b_{1}-\frac{a_{2}}{a_{1}}\right| \eta^{+^{2}}+\eta^{+^{4}} .
\end{aligned}
$$

Therefore, (3.3) and (3.4) are satisfied simultaneously if $M_{1} \tau^{2}+M_{2} \tau<M_{3}$ holds. Hence, Nyquist criterion holds for $0 \leq \tau \leq \tau^{+}$, where $\tau^{+}=\frac{1}{2 M_{1}}\left(-M_{2}+\sqrt{M_{2}^{2}+4 M_{1} M_{3}}\right)$ is the maximum length of the delay for which the stability of the bifurcating limit cycle is preserved. 


\begin{tabular}{|c|c|c|c|c|}
\hline Parameters & Descriptions & Values & Units & Sources \\
\hline$\lambda$ & Recruitment rate of uninfected cell & 10 & cells $\mathrm{ml}^{-1}$ day $^{-1}$ & {$[10,11]$} \\
\hline$\beta_{1}$ & Virus-to-cell infection rate & 0.01 & ml virion ${ }^{-1}$ day $^{-1}$ & {$[10,11]$} \\
\hline$\beta_{2}$ & Cell-to-cell infection rate & 0.01 & ml cell ${ }^{-1}$ day $^{-1}$ & {$[24]$} \\
\hline$d_{1}$ & Death rate of uninfected cell & 0.01 & day $^{-1}$ & [7] \\
\hline$d_{2}$ & Death rate of infected cell & 1 & day $^{-1}$ & [7] \\
\hline$d_{3}$ & Death rate of virion & 1 & day $^{-1}$ & {$[10,11]$} \\
\hline$d_{4}$ & Death rate of $B$ cell & 0.3 & day $^{-1}$ & {$[37]$} \\
\hline$\alpha$ & Cure rate of infected cell & 0.01 & day $^{-1}$ & [38] \\
\hline$k$ & Production rate of virion & 2.9 & virions cell ${ }^{-1}$ day $^{-1}$ & [7] \\
\hline$p$ & Neutralization rate of virion by $B$ cell & 0.1 & $\mathrm{ml} \mathrm{cell}^{-1}$ day $^{-1}$ & {$[39]$} \\
\hline$c$ & Development rate of $B$ cell & 0.1 & ml virion ${ }^{-1}$ day $^{-1}$ & [37] \\
\hline
\end{tabular}

Table 1: The list of parameter values for numerical simulations for the model system (2.1)

\section{NUMERICAL SimUlations AND Discussions}

In this section, we present several numerical illustrations to analyze the effect of time delay in the generation of $B$ cells, in addition to investigating the effect of the development rate of $B$ cells, on the dynamical behavior of the model system (2.1). In order to perform the numerical simulation, we chose the parameter values given in Table 1 and made use of MatLab ${ }^{\circledR}$. We first demonstrate the global stability of the boundary equilibria of the system (2.1) through numerical simulation, by considering three different initial conditions, namely, $i c_{1}:=(80,5,2,50), i c_{2}:=(50,2,0.5,80)$ and $i c_{3}:=(30,8,6,70)$ each for three different immune delays, namely, $\tau=6,12,25$. The various trajectories of uninfected hepatocytes (Figs. 1a and 2a), infected hepatocytes (Figs. 1b and 2b), virions (Figs. 1c and 2c) and $B$ cells (Figs. 1d and 2d) for three different initial conditions and three different time delays are presented as follows:

- Blue lines : $i c_{1}(80,5,2,50)$,

- Red lines : $i c_{2}(50,2,0.5,80)$,

- Green lines : $i c_{3}(30,8,6,70)$,

- Solid lines : $\tau=6$,

- Dashed lines : $\tau=12$,

- Dotted lines : $\tau=25$.

In order to illustrate the case $R_{0}<1$, we choose the parameter values listed in Table 1 , except $\lambda=1[10,11], \beta_{2}=0.001[24], d_{3}=6[7,40]$, which correspond to $R_{0}=0.5775$. This scenario 
is presented in Fig. 1, which shows that the uninfected hepatocytes (Fig. 1a) with three different initial levels, increase gradually and then finally stabilize at the level $T=100$, whereas the infected hepatocytes (Fig. 1b) as well as virions (Fig. 1c) gradually decrease and eventually converge to zero. The behavior of $B$ cells (Fig. 1d) depends on the initial condition at the beginning, but after a period of time, it also converges to zero. This simulation also indicates that the variation of immune delay does not in any way affect the trajectories of uninfected and infected hepatocytes as well as virion population (solid, dashed and dotted lines merged on same path in Figs. 1a, 1b and 1c), but the slow convergence of $B$ cells (solid lines of all colors converging to zero earlier than other patterned lines of corresponding colors in Fig. 1d) occurs due to increase in value of the immune delay. This suggests that the infection-free equilibrium $E_{0}(100,0,0,0)$ is globally asymptotically stable for any time delay in the generation of $B$ cells, which supports the theoretical result in Theorem 1.

In order to study the case $R_{H}<1<R_{0}$, we choose $\lambda=1[10,11]$ with the other parameter values being the ones as in Table 1 , which correspond to $R_{0}=3.8613<1+\frac{d_{2}}{\alpha}(=101)$ and $R_{H}=$ 0.7731. This scenario is presented in Fig. 2. This figure shows that the uninfected hepatocytes (Fig. 2a), infected hepatocytes (Fig. 2b) and virions (Fig. 2c) starting with three different initial positions are oscillating for a period of time and then finally converge to their corresponding stabilized levels ( $T=25.8974, I=0.7410, V=2.1489$ ). The trajectories of the corresponding populations follow the similar oscillatory behavior in all cases with the larger cycle (slower convergence) being observed due to increase in immune delay. The $B$ cell (Fig. 2d) after starting with different positions changes its behavior depending upon the initial conditions and then suddenly falls down to zero level. It is also observed that $B$ cells take more time to reach the zero stabilized level due to increase in immune delay. This simulation indicates that the process of $B$ cells generation will not be continued without a large amount of viral load and enough infection. This suggests that the immune-free equilibrium $E_{1}(25.8974,0.7410,2.1489,0)$ is globally asymptotically stable for any time delay in the generation of $B$ cells, which is obtained theoretically in Theorem 2 .

Furthermore, we perform numerical simulation to show the Hopf bifurcation and stability switches occurring at the interior equilibrium $E^{*}$ as the immune delay $\tau$ increases. For this purpose, we choose all the parameter values listed in Table 1 . The corresponding $R_{H}=7.7312>1$ and $E^{*}=(76.2402,9.2375,3,79.2967)$. We compute the roots of the equation (2.11) and obtain two positive real roots, namely, $\gamma_{1}=0.1235$ and $\gamma_{2}=0.1082$, which satisfy the condition of Theorem 3 . Therefore, using the formula (2.13), we calculate $\tau_{n}^{(j)}, n=1,2 ; j=0,1,2, \ldots$ as follows

$$
\begin{gathered}
\tau_{1}^{(0)}=5.7944, \tau_{2}^{(0)}=7.4298, \tau_{1}^{(1)}=23.6734, \\
\tau_{2}^{(1)}=26.5270, \tau_{1}^{(2)}=41.5524, \tau_{2}^{(2)}=45.6242
\end{gathered}
$$


with the value of $\tau_{n}^{(j)}$ for $j \geq 3$ being calculated similarly. Therefore we get $\tau_{0}=\tau_{1}^{(0)}=5.7944$ and $G^{\prime}\left(\omega_{0}^{2}\right)=1.7072>0$, which satisfies the existence condition of Hopf bifurcation in Theorem 3 . We plot the densities (Figs. 3a-8a) of the four model populations against time and the phase portraits (Figs. 3b-8b) of the trajectories of uninfected and infected hepatocytes as well as virion population for various time delays $(\tau)$. It is clearly noticed in Fig. 3 that $E^{*}(76.2402,9.2375,3,79.2967)$ is locally asymptotically stable when $\tau=5<\tau_{0}$. From the numerical simulation, it can be observed that $E^{*}$ is locally asymptotically stable when $\tau \in\left[0, \tau_{0}\right)$, which is obtained theoretically in Theorem 3. Moreover, by Theorem 3, when $\tau$ is increased past the critical value $\tau_{0}$, a Hopf bifurcation occurs at $\tau=\tau_{0}$. The result for $\tau=6>\tau_{0}$ is shown in Fig. 4, which implies that $E^{*}$ becomes unstable and consequently a bifurcating periodic solution exists for $\tau \in\left(\tau_{0}, \tau_{2}^{(0)}\right)$. Again, when $\tau$ crosses another critical value $\tau_{2}^{(0)}, E^{*}$ regains local asymptotical stability which is presented for a particular value $\tau=14$ in Fig. 5. This shows that $E^{*}$ is asymptotically stable again for $\tau \in\left(\tau_{2}^{(0)}, \tau_{1}^{(1)}\right)$. For the case $\tau \in\left(\tau_{1}^{(1)}, \tau_{2}^{(1)}\right), E^{*}$ losses stability and a periodic oscillation exists around the equilibrium $E^{*}$ which is exhibited for $\tau=25$ in Fig. 6. If $\tau$ is increased and exceeds $\tau_{2}^{(1)}$, then the interior equilibrium $E^{*}$ becomes stable again, which is illustrated with $\tau=35 \in\left(\tau_{2}^{(1)}, \tau_{1}^{(2)}\right)$ in Fig. 7. Further, we simulate the system for a large value of $\tau$, namely, $\tau=43 \in\left(\tau_{1}^{(2)}, \tau_{2}^{(2)}\right)$, which is demonstrated in Fig. 8, showing that the stability switch occurs again with a periodic oscillation of the populations. In the same way, when the bifurcation parameter $\tau$ is increased and passes the critical bifurcation values $\tau_{0}, \tau_{2}^{(0)}, \tau_{1}^{(1)}, \tau_{2}^{(1)}, \tau_{1}^{(2)}, \tau_{2}^{(2)}$ and so on; stability of the interior equilibrium $E^{*}$ of the system (2.1) changes from stable to unstable, Hopf bifurcation occurs at these critical values and then regains asymptotic stability from unstable behavior successively. From this numerical discussion, we can conclude that Hopf bifurcation and stability switches occur at the critical values of the bifurcation parameter, which are $\tau_{1}^{(j)}$ and $\tau_{2}^{(j)}, j=0,1,2, \ldots$ Therefore, combining the numerical results obtained, one can finally conclude that the interior equilibrium $E^{*}(76.2402,9.2375,3,79.2967)$ is locally asymptotically stable for $\tau \in\left[0, \tau_{0}\right) \bigcup\left[\bigcup_{j=0}^{\infty}\left(\tau_{2}^{(j)}, \tau_{1}^{(j+1)}\right)\right]$ with $\tau_{2}^{(j)}<\tau_{1}^{(j+1)}, j=0,1,2, \ldots$

In the preceding discussion, we have shown that the time delay in generation of $B$ cells has a significant influence on the dynamical behavior of the system. Moreover, as $B$ cells are directly involved in the neutralization of the virions, so the viral infection can be affected depending on the development rate of $B$ cells as well. We therefore investigate (in Fig. 9), the effect of the development rate $(c)$ of $B$ cells on the dynamics of uninfected hepatocytes (Fig. 9a), infected hepatocytes (Fig. 9b) and viral load (Fig. 9c) as well as antibody response (Fig. 9d). For this, the numerical simulation is performed for five different values of $c$, namely, $c=0.01,0.03,0.07,0.1,0.5$ with the other parameters values being retained as in Table 1. It is observed from Fig. 9 that when $B$ cells are 
generated very slowly (in case of $c=0.01$ ), the viral load as well as infected hepatocytes increase to a very high level, which results in a decrease of the uninfected hepatocytes to a very low level resulting in the antibody not responding anymore. Moreover, a slight increase in the development rate of $B$ cells, raises the uninfected hepatocytes highly with a significant decrease in virions density. However, the changes in density of infected hepatocytes with the development rate of $B$ cells is relatively very small. For a certain range of $c$ (for example $c=0.07,0.1$ ), disturbance in the stability of the equilibrium and subsequent occurrence of bifurcating periodic orbits are noticed. Finally, one can observe from the expression of $R_{0}$ and $R_{H}$ that $R_{H}$ is positively correlated with $c$, but $c$ does not affect $R_{0}$. Therefore complete cure of infection for a patient is not possible by only magnifying the development rate of $B$ cells. However, a high antigenic stimulation in the generation of $B$ cells is beneficial for uninfected hepatocytes.

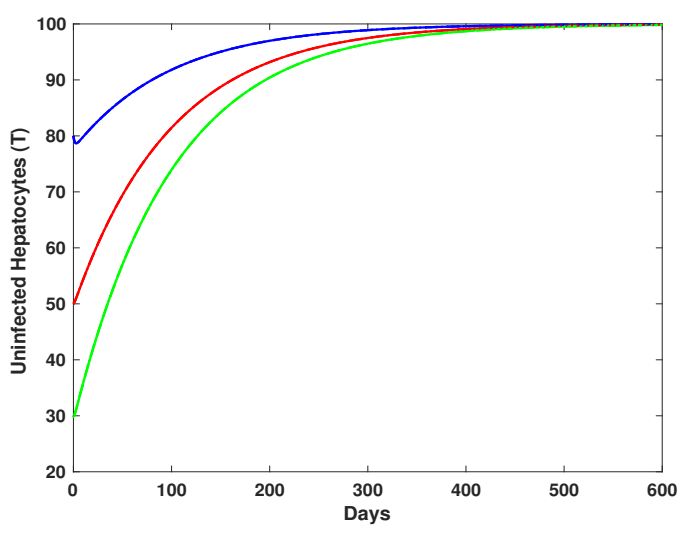

(a)

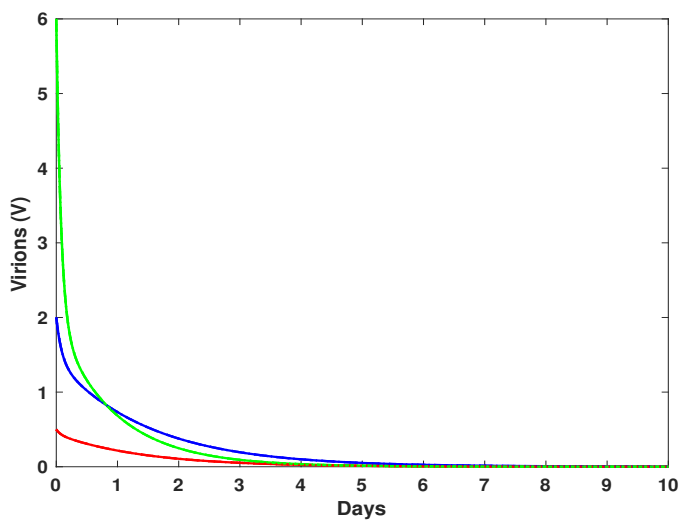

(c)

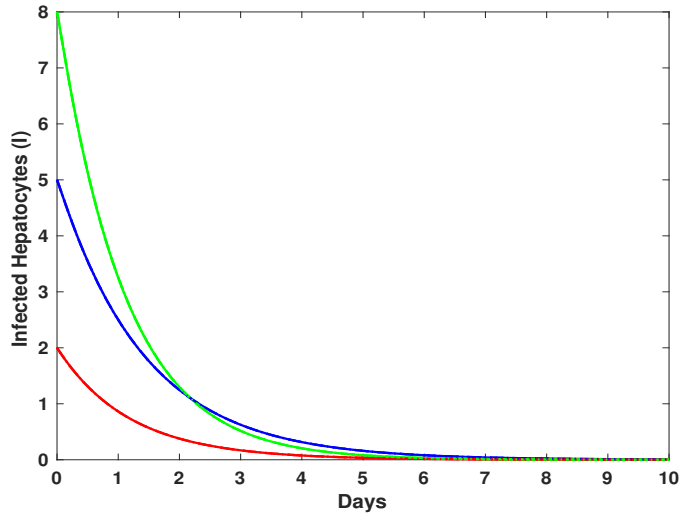

(b)

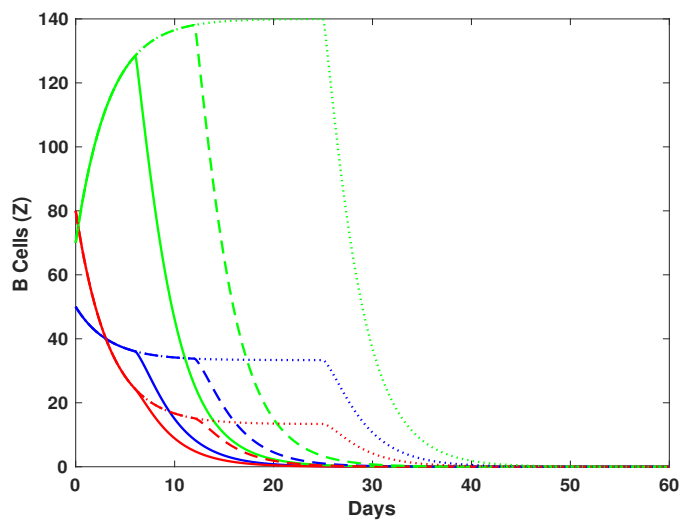

(d)

Figure 1: The trajectories of the four populations of the system (2.1) with three different initial conditions $i c_{1}, i c_{2}, i c_{3}$ and three immune delays $\tau=6,12,25$ in case of $R_{0}<1$. Blue lines : $i c_{1}$, red lines : $i c_{2}$, green lines : $i c_{3}$, solid lines : $\tau=6$, dashed lines : $\tau=12$, dotted lines : $\tau=25$. 


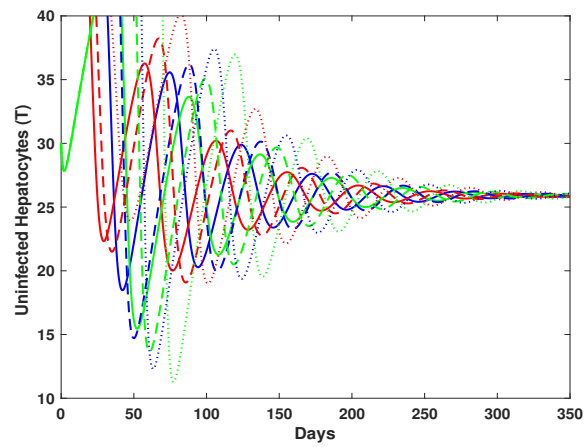

(a)

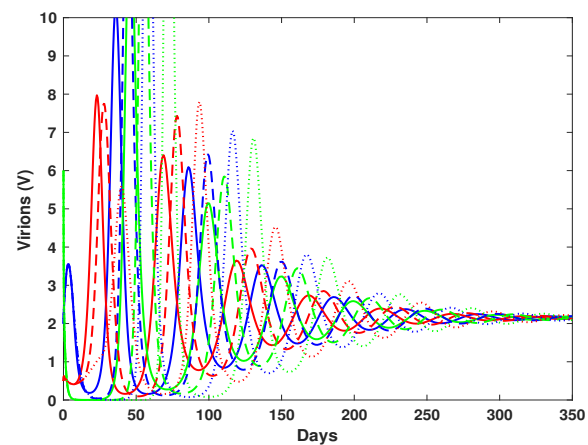

(c)

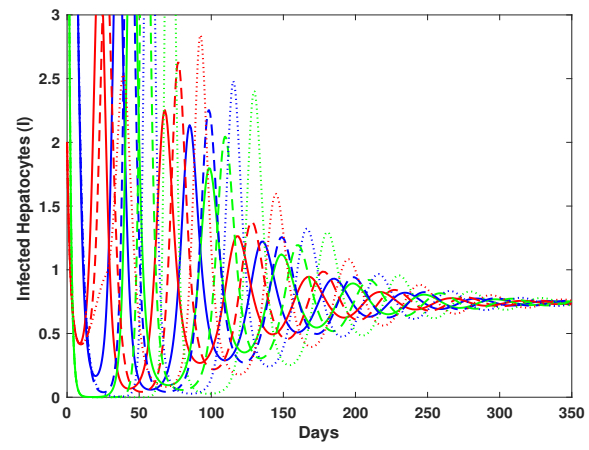

(b)

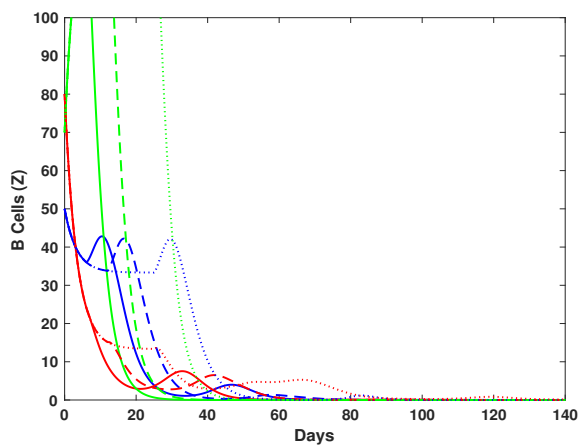

(d)

Figure 2: The trajectories of the four populations of the system (2.1) with three different initial conditions $i c_{1}, i c_{2}, i c_{3}$ and three immune delays $\tau=6,12,25$ in case of $R_{H}<1<R_{0}$. Blue lines : $i c_{1}$, red lines : $i c_{2}$, green lines : $i c_{3}$, solid lines : $\tau=6$, dashed lines : $\tau=12$, dotted lines : $\tau=25$.

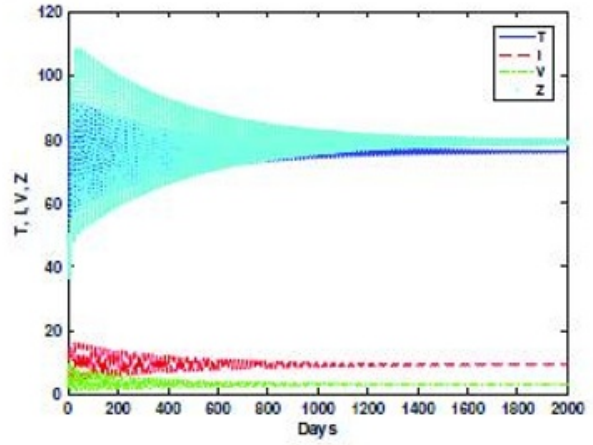

(a)

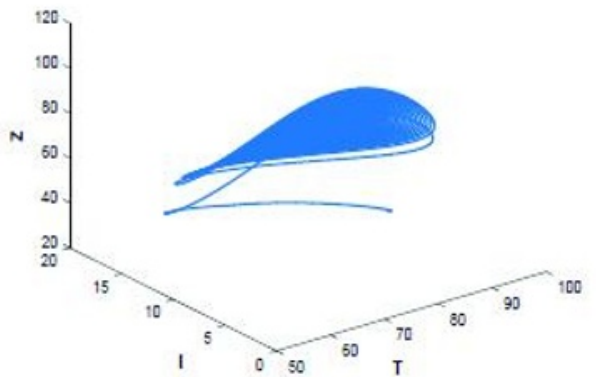

(b)

Figure 3: The time histories and the phase portraits of trajectories shows the local asymptotic stability of $E^{*}$ for immune delay $\tau=5<\tau_{0}$.

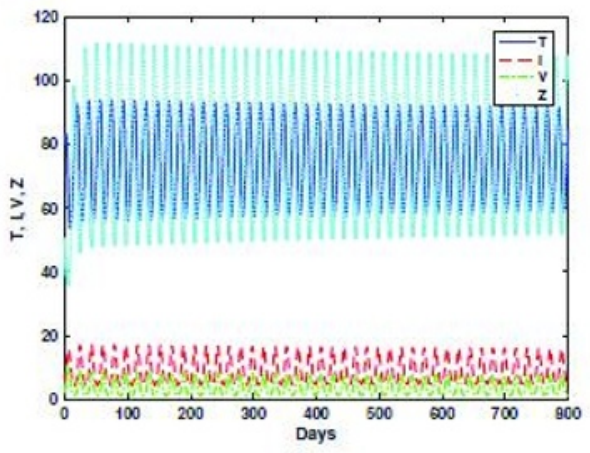

(a)

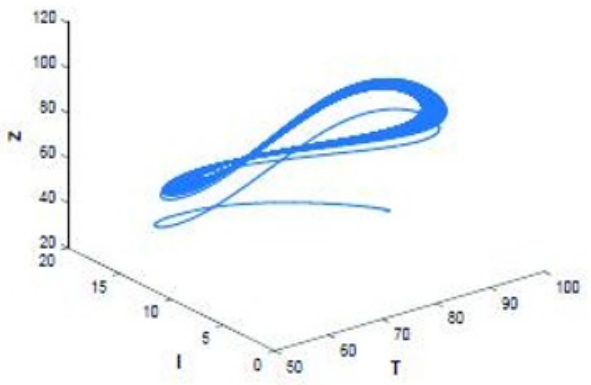

(b)

Figure 4: The time histories and the phase portraits of trajectories shows the bifurcating periodic orbits around $E^{*}$ in case of immune delay $\tau=6 \in\left(\tau_{0}, \tau_{2}^{(0)}\right)$. 


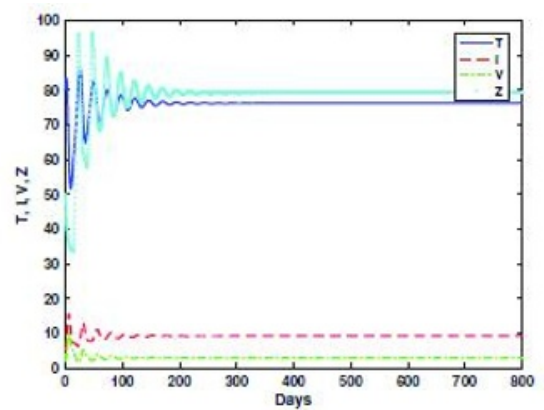

(a)

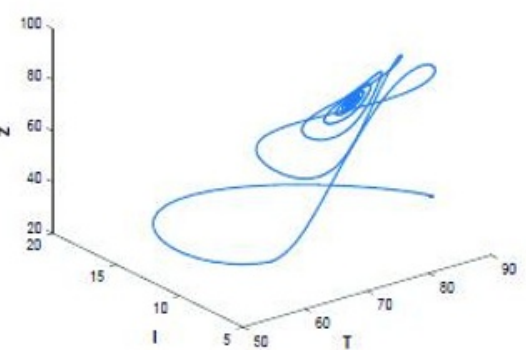

(b)

Figure 5: The time histories and the phase portraits of trajectories shows the local asymptotic stability of $E^{*}$ for immune delay $\tau=14 \in\left(\tau_{2}^{(0)}, \tau_{1}^{(1)}\right)$.

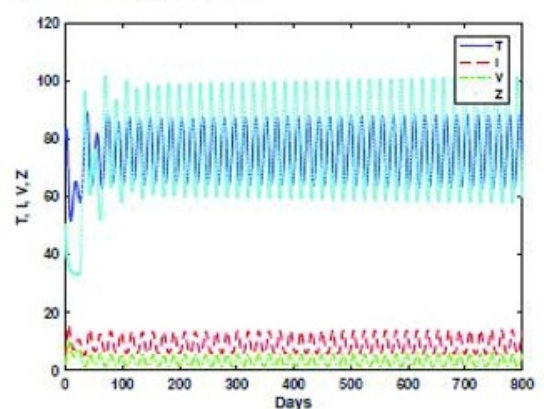

(a)

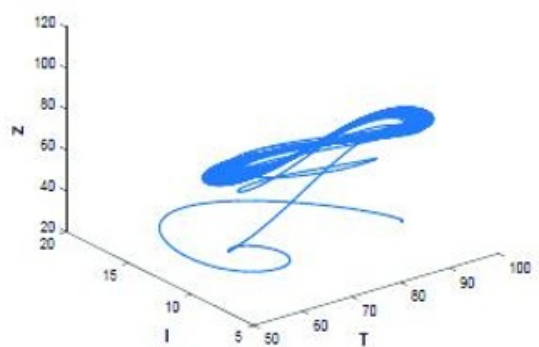

(b)

Figure 6: The time histories and the phase portraits of trajectories shows the existence of a periodic oscillation around $E^{*}$ for immune delay $\tau=25 \in\left(\tau_{1}^{(1)}, \tau_{2}^{(1)}\right)$.

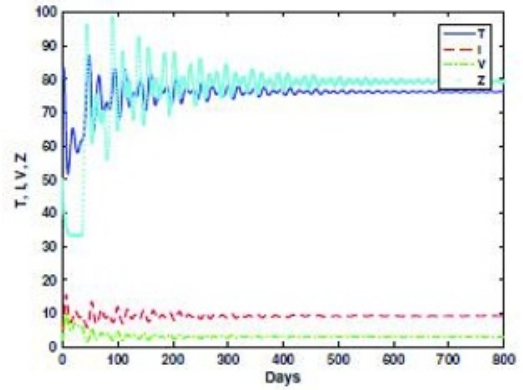

(a)

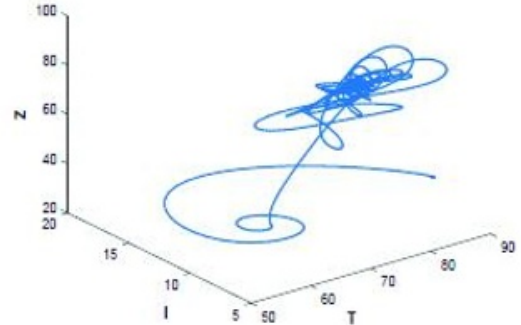

(b)

Figure 7: The time histories and the phase portraits of trajectories shows the local asymptotic stability of $E^{*}$ for immune delay $\tau=35 \in\left(\tau_{i}^{(1)}, \tau_{1}^{(2)}\right)$.

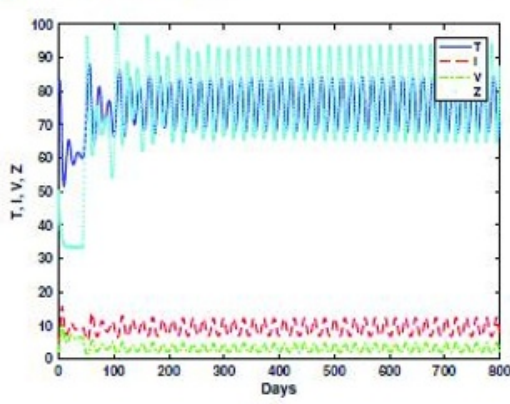

(a)

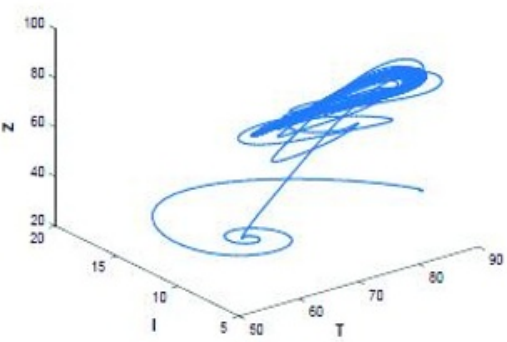

(b)

Figure 8: The time histories and the phase portraits of trajectories shows the existence of a periodic bifurcation around $E^{*}$ for immune delay $\tau=43 \in\left(\tau_{1}^{(2)}, \tau_{2}^{(2)}\right)$. 


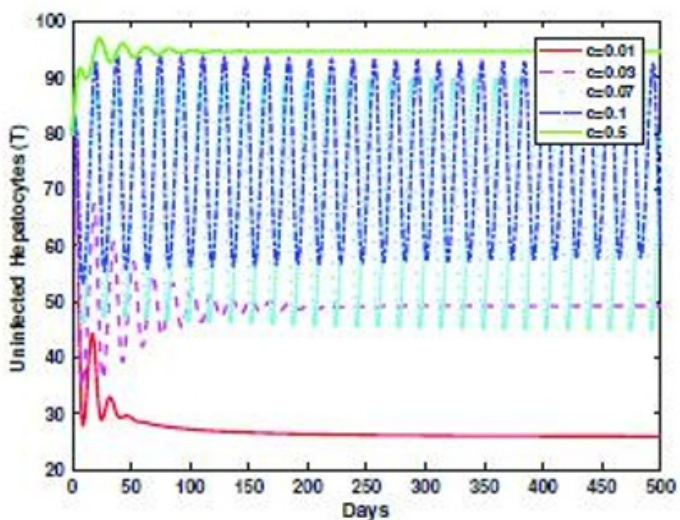

(a)

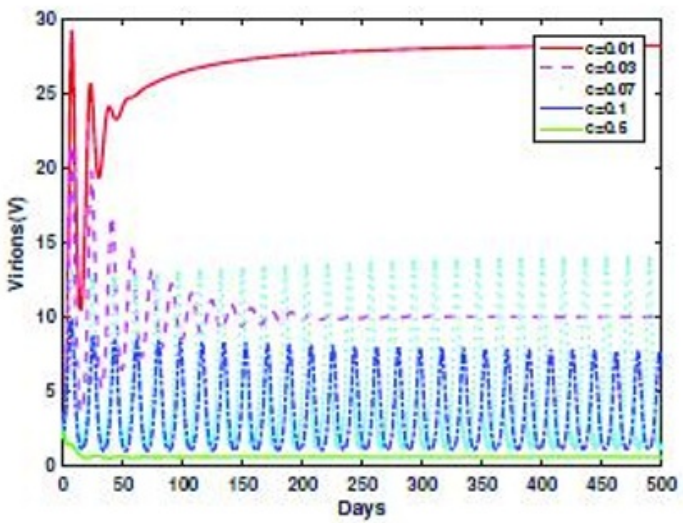

(c)

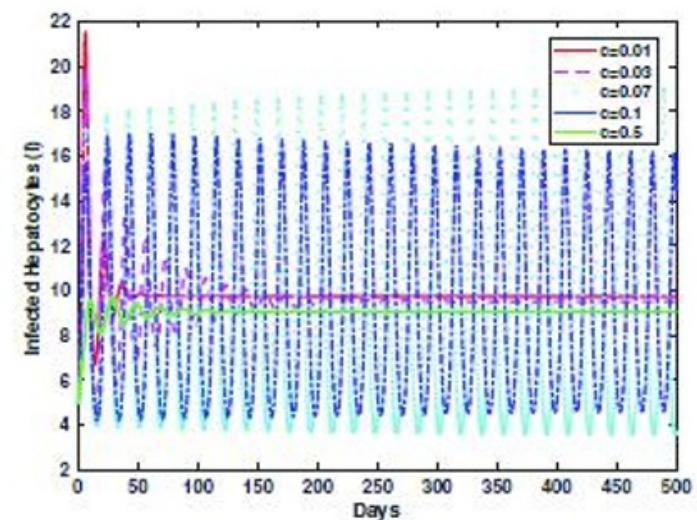

(b)

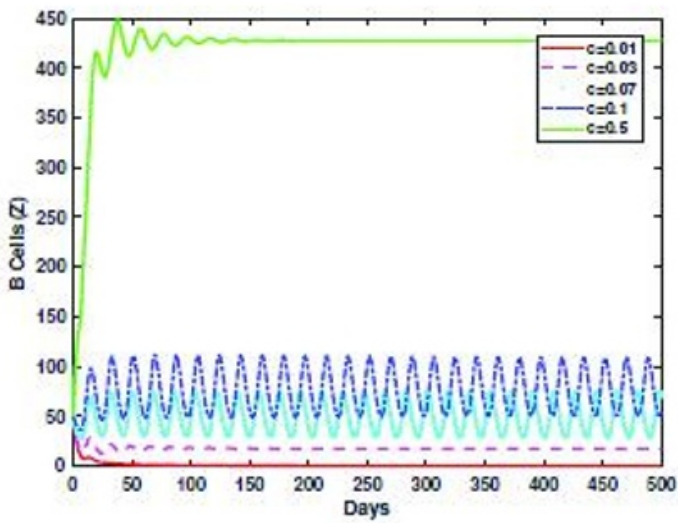

(d)

Figure 9: The effect of $c$ (development rate of $B$ cells) on the dynamical behavior of the system (2.1).

\section{CONCLUSiONS}

In this paper, we analyzed HCV dynamics considering both viral and cellular transmissions with the effect of humoral immune delay. The model also included the conversion of infected hepatocytes into uninfected ones through the non-cytolytic process. The feasibility of the model has been justified by establishing the positivity and boundedness of the solution. We have investigated that the boundary equilibria, namely, infection-free and immune-free equilibrium are locally as well as globally asymptotically stable under the conditions on the basic reproduction number as well as the humoral immune reproduction number. The infection-free and immune-free equilibrium are globally asymptotically stable if $R_{0} \leq 1$ and $R_{H} \leq 1<R_{0} \leq 1+\frac{d_{2}}{\alpha}$ respectively. The model system undergoes a Hopf bifurcation from the interior equilibrium when the bifurcation parameter crosses the critical values. The numerical results demonstrate the existence of stability switches and bifurcating periodic solutions due to increase in the immune delay. 
The simulation showed that the development rate of $B$ cells drives the system from stable to unstable and then from unstable to stable again. The findings suggested that a small increment in the development rate of $B$ cells significantly increases the uninfected hepatocytes being neutralizing the virions. Therefore, a high antigenic stimulation in the generation of $B$ cells is beneficial for uninfected hepatocytes. However complete cure from infection not possible by only magnifying the development rate of $B$ cells.

\section{ACKNOWLEDGEMENT}

The first author is grateful to Indian Institute of Technology Guwahati for the financial support provided to pursue his Ph.D.

\section{REFERENCES}

1. World Health Organization, Global hepatitis report 2017. World health organization: Geneva, Switzerland, 2017.

2. A. Dev, V. Sundararajan, and W. Sievert. Ethnic and cultural determinants influence risk assessment for hepatitis C acquisition, Journal of Gastroenterology and Hepatology, 19(7) (2004), 792-798.

3. H. R. Rosen, Chronic hepatitis C unfection, The New England Journal of Medicine, 364(25) (2011), $2429-2438$.

4. M. Lechmann and T. J. Liang, Vaccine development for hepatitis C, Seminars in liver disease, 20(2) (2000), 211-226.

5. A. U. Neumann, N. P. Lam, H. Dahari, D. R. Gretch, T. E. Wiley, T. J. Layden, and A. S. Perelson. Hepatitis $\mathrm{C}$ viral dynamics in vivo and the antiviral efficacy of interferon- $\alpha$ therapy, Science, 282(5386) (1998), 103-107.

6. N. M. Dixit, J. E. Layden-Almer, T. J. Layden, and A. S. Perelson, Modelling how ribavirin improves interferon response rates in hepatitis C virus infection, Nature, 432(7019) (2004), 922-924.

7. H. Dahari, A. Lo, R. M. Ribeiro, and A. S. Perelson, Modeling hepatitis C virus dynamics: Liver regeneration and critical drug efficacy, Journal of Theoretical Biology, 247(2) (2007), 371-381.

8. H. Dahari, R. M. Ribeiro, and A. S. Perelson, Triphasic decline of hepatitis C virus RNA during antiviral therapy, Hepatology, 46(1) (2007), 16-21.

9. A. S. Perelson, E. Herrmann, F. Micol, and S. Zeuzem, New kinetic models for the hepatitis C Virus, Hepatology, 42(4) (2005), 749-754.

10. D. Wodarz, Hepatitis C virus dynamics and pathology: the role of CTL and antibody responses, Journal of General Virology, 84(7) (2003), 1743-1750. 
11. D. Wodarz, Mathematical models of immune effector responses to viral infections: Virus control versus the development of pathology, Journal of computational and applied mathematics, 184(1) (2005), 301319.

12. J. Li, K. Men, Y. Yang, and D. Li, Dynamical analysis on a chronic hepatitis C virus infection model with immune response, Journal of Theoretical Biology, 365 (2015), 337-346.

13. H. Dahari, M. Major, X. Zhang, K. Mihalik, C. M. Rice, A. S. Perelson, S. M. Feinstone, and A. U. Neumann, Mathematical modeling of primary hepatitis $\mathrm{C}$ infection: Noncytolytic clearance and early blockage of virion production, Gastroenterology, 128(4) (2005), 1056-1066.

14. T. C. Reluga, H. Dahari, and A. S. Perelson, Analysis of hepatitis C virus infection models with hepatocyte homeostasis, SIAM Journal on Applied Mathematics, 69(4) (2009), 999-1023.

15. J. M. Timpe, Z. Stamataki, A. Jennings, K. Hu, M. J. Farquhar, H. J. Harris, A. Schwarz, I. Desombere, G. L. Roels, P. Balfe, and J. A. McKeating, Hepatitis C virus cell-cell transmission in hepatoma cells in the presence of neutralizing antibodies, Hepatology, 47(1) (2008), 17-24.

16. A. Mojaver and H. Kheiri, Dynamical analysis of a class of hepatitis $\mathrm{C}$ virus infection models with application of optimal control, International Journal of Biomathematics, 9(3) (2016), 1650038.

17. Y. Zhao and Z. Xu, Global dynamics for a delayed hepatitis $\mathrm{C}$ virus infection model, Electronic Journal of Differential Equations, 2014(132) (2014), 1-18.

18. D. Wu, Y. Su, and D. Sun, Stability properties and Hopf bifurcation for a Hepatitis B infection model with exposed state and humoral immunity-response delay, Communications in Mathematical Biology and Neuroscience, 2015, 2015.

19. T. Wang, Z. Hu, and F. Liao, Stability and Hopf bifurcation for a virus infection model with delayed humoral immunity response, Journal of Mathematical Analysis and Applications, 411(1) (2014), 63-74.

20. Y. Yang, L. Zou, and S. Ruan, Global dynamics of a delayed within-host viral infection model with both virus-to-cell and cell-to-cell transmissions, Mathematical biosciences, 270 (2015), 183-191.

21. Q. Hu, Z. Hu, and F. Liao, Stability and Hopf bifurcation in a HIV-1 infection model with delays and logistic growth, Mathematics and Computers in Simulation, 128 (2016), 26-41.

22. J. Wang, M. Guo, X. Liu, and Z. Zhao, Threshold dynamics of HIV-1 virus model with cell-to-cell transmission, cell-mediated immune responses and distributed delay, Applied Mathematics and Computation, 291 (2016), 149-161.

23. S. S. Chen, C. Y. Cheng, and Y. Takeuchi, Stability analysis in delayed within-host viral dynamics with both viral and cellular infections, Journal of Mathematical Analysis and Applications, 442(2) (2016), 642-672.

24. S. Pan and S. P. Chakrabarty, Threshold dynamics of HCV model with cell-to-cell transmission and a non-cytolytic cure in the presence of humoral immunity, Communications in Nonlinear Science and 
Numerical Simulation, 61 (2018), 180-197.

25. K. F. Wang, W. D. Wang, H. Y. Pang, and X. N. Liu, Complex dynamic behavior in a viral model with delayed immune response, Physica D: Nonlinear Phenomena, 226(2) (2007), 197-208.

26. J. K. Hale and S. M. V. Lunel, Introduction to functional differential equations, Springer Science and Business Media, 99 (2013).

27. H. R. Thieme, Mathematics in population biology, Princeton University Press, Princeton, 2003.

28. X. Yang, L. S. Chen, and J. F. Chen, Permanence and positive periodic solution for single-species nonautonomous delay diffusive model, Computers and Mathematics with Applications, 32(4) (1996), 109-116.

29. P. Driessche and J. Watmough, Reproduction numbers and sub-threshold endemic equilibria for compartmental models of disease transmission, Mathematical Biosciences, 180(1-2) (2002), 29-48.

30. J. M. Heffernan, R. J. Smith, and L. M. Wahl, Perspectives on the basic reproductive ratio, Journal of the Royal Society Interface, 2(4) (2005), 281-293.

31. H. I. Freedman and V. S. H. Rao, The trade-off between mutual interference and time lags in predatorprey systems, Bulletin of Mathematical Biology, 45(6) (1983), 991-1004.

32. J. E. Marsden and M. McCracken, The Hopf bifurcation and its applications, Springer Science and Business Media, 19 (2012).

33. X. Li and J. Wei, On the zeros of a fourth degree exponential polynomial with applications to a neural network model with delays, Chaos, Solitons \& Fractals, 26(2) (2005), 519-526.

34. H. Miao, Z. Teng, C. Kang, and A. Muhammadhaji, Stability analysis of a virus infection model with humoral immunity response and two time delays, Mathematical Methods in the Applied Sciences, 39(12) (2016), 3434-3449.

35. L. H. Erbe, H. I. Freedman, and V. S. H. Rao, Three-species food-chain models with mutual interference and time delays, Mathematical Biosciences, 80(1) (1986), 57-80.

36. H. Nyquist, Regeneration theory, Bell Labs Technical Journal, 11(1) (1932), 126-147.

37. J. Reyes-Silveyra and A. R. Mikler, Modeling immune response and its effect on infectious disease outbreak dynamics, Theoretical Biology and Medical Modelling, 13(1) 10, 2016.

38. B. Dubey, P. Dubey, and U. S. Dubey, Modeling the intracellular pathogen-immune interaction with cure rate, Communications in Nonlinear Science and Numerical Simulation, 38 (2016), 72-90.

39. A. Murase, T. Sasaki, and T. Kajiwara, Stability analysis of pathogen-immune interaction dynamics, Journal of Mathematical Biology, 51(3) (2005), 247-267.

40. S. Banerjee, R. Keval, and S. Gakkhar, Modeling the dynamics of hepatitis C virus with combined antiviral drug therapy: Interferon and ribavirin, Mathematical Biosciences, 245(2) (2013), 235-248. 